\title{
The electronic structures of the atoms
}

\section{La structure électronique des atomes}

Jozsef Garai ${ }^{\text {a) }}$

University of Debrecen, College of Engineering, Department of Civil Engineering, Ótemetö u. 2-4, 4028 Debrecen, Hungary

Abstract: Based on the uniformity and the stability of the atoms it is concluded that the point charge nature of the electron in the vicinity of the nucleus is unsustainable. It is shown that uniform spherical surface charge "electron" around the nucleus can explain not only the observed uniformity and stability of the atoms but also successfully describes the electromagnetic radiation of the atom, gives physical explanation for the quantized nature of the angular momentum, and explains the wave particle duality of matter. Additionally, the model gives the correct zero angular momentum at the ground state and the right ionization energy, which features were not explained by previous models. The presented physical explanations for the atomic scale observations of matter does not violate the laws of classical physics indicating that these laws should remain valid at atomic scale.

Résumé: Selon la base de l'uniformité et la stabilité des atomes, on peut conclure que le modèle de charge ponctuelle de l'électron dans le voisinage du noyau est insoutenable. Il est montré que le modéle de charge de surface sphérique uniforme autour du noyau peut expliquer non seulement l'uniformité et la stabilité observées des atomes, mais aussi il décrit avec succès le radiation électromagnétique de l'atome. Il donne également l'explication physique de la nature quantifiée du moment angulaire, et explique la dualité onde-particule de la matière. Le modèle 
offre des explications pour les observations à l'échelle atomique de la matière, sans violer les lois de la physique classique, indiquant que ces lois devraient rester valables à l'échelle atomique.

Key words: Structure of the atom; Atom model; Electronic structure of the atom; Bohr's model; Cloud model; Wave-particle duality; Quantum mechanics; Quantum physics; Photon emission absorption a)jozsef.garai@fiu.edu 


\section{INTRODUCTION}

The first modern version of atomic model was proposed by Rutherford ${ }^{1}$. Based on the scattering experiments of Hans Geiger and Ernest Marsden it was concluded that, most of the space inside the atom is empty, there is a small, heavy positively charged core (nucleus) inside the atom, which contains almost the entire mass of the atom ${ }^{1}$. The nucleus also contains neutrons, which has almost the same mass as the proton but has no charge ${ }^{2,3}$. The negatively charged electrons are orbiting around the nucleus, compensating the positive charge of the protons resulting in a charge neutrality of the atoms. In order to comply with spectroscopic observations Bohr was suggesting that the angular momentum of the electrons orbiting around the nucleus have certain discrete values ${ }^{4}$. Bohr's planetary model successfully explains the main emission lines of the hydrogen ${ }^{5}$, derives the correct values for the Rydberg constant ${ }^{6}$, the radius of the hydrogen atom and the ionization energy ${ }^{4}$. Despite this enormous success the model has many shortcomings ${ }^{7}$. Sommerfeld ${ }^{8}$ made an adjustment to Bohr's original model by allowing elliptical orbits for the electrons. The Bohr-Sommerfeld model correctly calculates the fine structure splitting of hydrogen but it has been shown that the theoretical explanation for the splitting is the spin-orbit interaction instead of the elliptical orbits. The discovery of the waveparticle duality of matter ${ }^{9}$, the uncertainty principle ${ }^{10,11}$ and the wave nature of objects on the atomic scale ${ }^{12}$ led to the development of quantum mechanics ${ }^{13-17}$. Assigning probability to the wave function ${ }^{18,19}$ allows calculating the positions of the electron/s. Thus the position of the electron cannot be defined precisely and only the probability of finding in a certain region can be determined. The completely quantum mechanical treatment of the atomic structure reproduces all of the emission lines of the Hydrogen atom with no discrepancy. Despite this enormous 
success of the quantum cloud model of the atoms, the model still has few shortcomings, which are discussed here.

\section{THE SHORTCOMINGS OF PREVIOUS ATOM MODELS}

The atoms built up from two oppositely charged principal components, the positively charged nucleus and the negatively charged electrons. Based on Rutherford's experiments the description of the nucleus at atomic level is considered to be settled and not discussed here. However, the electronic structure of the atom is not completely understood and its structure is still debated.

Bohr described the electron as a point particle, while quantum mechanics treats the electron as cloud with no certain position. Bohr uses classical physics while quantum mechanics describes the electron by the wave function. Both the Bohr and the cloud models are able to explain many atomic phenomenons correctly, indicating that many of their assumptions are valid. The main features of the atoms and how previous models comply with these features are discussed in detail.

\section{1./ Classical physics}

Bohr postulates that the Coulomb attraction between the electron and the nucleus is valid (i) and the orbiting electron around the proton obeys the laws of classical mechanics (ii). He also postulates that the law of electromagnetism does not apply at atomic scale because electron with a constant acceleration moving in an allowed orbit does not radiate electromagnetic energy (iii). His fourth postulates is that radiation is emitted only, when an atoms makes transition between stationary states (iiii). No explanation is given why certain laws of electromagnetism (iii; iiii) 
are invalid at atomic scale. Selectively discrediting parts of the laws of classical physics, especially those which are not conformed to the "given model" is not justifiable.

\section{2./ Point charge nature of the electron at atomic scale}

The Bohr's model assumes that the electron is a point charge in the atom. The cloud model also treats the electron as a point charge but suggest that this point charge is fuzzy and the position of the charge cannot be determined exactly.

In the time scale of the existence of the atom the orbit of a point charge electron is chaotic. This chaotic orbit of the electron sometimes should result in collision of the electron and the proton. Thus in the time scale of the universe no or very few stable atom should exist, which contradicts with the observed stability of the atoms.

\section{3./ The Bohr radius}

The derived radius for the Hydrogen atom is known as the Bohr's radius [ $\left.\mathrm{a}_{0}\right]$. This constant $\left[0.52917721067(12) \times 10^{-10} \mathrm{~m}\right.$.] is considered to be one of the most important one in physics ${ }^{21}$. Using Bohr's postulates made for his atomic model the radius can be derived from first principles. Interestingly, Bohr's derivation of the radius contradicts with his own postulates. $\mathrm{He}$ assumes that, without stating it, the mass center of the two bodies, proton and electron, are the same. This assumption is incorrect because does not take into account the reduced mass affect of the two body system (Fig. 1). Ignoring this effect introduces a small $(0.1 \%)$ error in the radius. More interestingly this "incorrectly" derived Bohr radius correlates perfectly with other fundamental constants. This agreement with the "incorrect" radius with other fundamental constant indicates that the mass center of the proton and the proton-electron system should be the same as it was assumed in Bohr calculation. 


\section{4./ Quantized angular momentum}

The relationship between the angular momentum and the Planck equation was derived by Nicholson $^{22}$. Bohr's postulated that the angular momentum of the electron [L] is quantized such as $L=m_{e} v r=n \hbar$, where $m_{e}$ is the mass of the electron, $v$ is the velocity of the orbiting electron, $r$ is the radius of the atom, $\mathrm{n}$ is a positive integer, and $\hbar=0.5 \mathrm{~h} / \pi$. Based on this assumption the correct values of the main spectra of hydrogen atom, and also other hydrogen-like atoms and ions, can be derived. Thus the quantized nature of the angular momentum in atomic scale can be considered as well established. However, there is a shortcoming of Bohr's postulate. The model predicts an incorrect value for the angular momentum of the electron at ground state $\mathrm{L}=\hbar$ instead of the observed $\mathrm{L}=0$.

\section{5./ Ionization energy of the Hydrogen atom}

The ionization energy is the quantity of energy that an isolated, gaseous atom in the ground electronic state must absorb to discharge an electron. The discharging of the electron requires removing the electron of the Hydrogen atom from its original position, distanced from the proton by the Bohr radius, to infinity. The required energy can be calculated as:

$$
I E=\int_{a_{o}}^{\infty} \frac{1}{4 \pi \varepsilon_{o}} \frac{e^{2}}{r^{2}} d r=\left[\frac{1}{4 \pi \varepsilon_{o}} \frac{e^{2}}{r}\right]_{r=a_{o}}^{r=\infty}=\frac{1}{4 \pi \varepsilon_{o}} \frac{e^{2}}{a_{o}}
$$

where $\mathrm{e}$ is the elementary charge, charge of the proton and electron, $\mathrm{r}$ is the distance between the proton and electron and $\varepsilon_{\mathrm{o}}$ is the permittivity of free space, which has the value ${ }^{23}$ $8.854187817 \ldots \times 10^{-12} \mathrm{C}^{2} / \mathrm{N}^{-1} \mathrm{~m}^{-2}$. Substituting the known physical constants into Eq. 1 gives the ionization energy $27.21 \mathrm{eV}$.

The ionization energy of the Hydrogen atom pops out nicely from the Bohr model. The calculated value (13.6057 eV) agrees well with experiments giving the currently accepted 
official value $13.59844 \mathrm{eV}^{24}$. This ionization energy of the Bohr model, and experiments is exactly half of the ionization energy calculated by Eq. 1. This discrepancy should be explained.

\section{6./ Emission and absorption of electromagnetic waves}

Bohr postulated that radiation is emitted only, when an atoms makes transition between stationary states. He describes the stationary states by different orbital radius, which is not supported by experiments. According to quantum mechanics, photon emission or absorption occurs, when the excitation or energy level is changing, which is very similar to Bohr's postulate. Currently there is no coherent physical model, which could explain the emission and absorption of photons and why the photon emission occurs only at transition between the energy states.

\section{7./ The wave nature of the matter}

The wave nature of the electron has been shown by the experiments of Davisson and Germer $^{12}$. This is the fundamental base of quantum mechanics, which use the wave function to describe all the physical phenomenon at atomic scale. The predictions of quantum mechanics are consistent with experiments. Thus the wave description of matter at atomic scale should be valid. However, no physical model for the wave nature of matter has been offered.

It is concluded that any atomic models should comply with and explaining the followings:

1./ laws of classical electromagnetism should not be selectively invalidated at atomic scale 2./ the stability and uniformity of the atoms, in the time frame of the existing universe, should be explained

3./ in the Hydrogen atom the mass center of the proton and the proton-electron system should be the same or the reduced mass radius should be used for the orbiting electron 
4./ the angular momentum of the electron should be zero at ground state

5./ the discrepancy between the ionization energy, calculated from the Bohr model, supported by experiments and from classical physics must be resolved

6./ the physics of the emission, occurring only when there is transition between the stationary energy levels, should be explained

7./ the physical explanation of the wave-particle duality nature of matter at atomic scale should be given.

\section{UNIFORM SURFACE CHARGE HALO DISTRIBUTION OF THE ELECTRONS}

Analyzing the listed problems with the previous atomic models it is suggested that the electron should have a uniform surface charge halo distribution at atomic scale. This atomic model is investigated in detail.

According to Coulombs law the electrostatic force (F) between two charges (q1 and q2) in a non-vector form is

$$
F=k \frac{q_{1} q_{2}}{r^{2}}
$$

where $\mathrm{k}$ is the Coulomb constant and $\mathrm{r}$ is the distance between the charges. The constant can also be given as:

$$
k=\frac{1}{4 \pi \varepsilon_{o}} .
$$

In case of the Hydrogen atom the charge of the proton and the electron is the elementary charge (e) and the distance between them in ground state is the Bohr radius $\left(a_{0}\right)$. The electrostatic force between the two charges is 


$$
F=\frac{1}{4 \pi \varepsilon_{o}} \frac{e^{2}}{a_{o}^{2}}=\frac{1}{\varepsilon_{o}} e \sigma_{e}\left(e_{o}\right)
$$

where

$$
\sigma_{e}\left(a_{o}\right)=\frac{e}{4 \pi a_{o}^{2}}
$$

is the surface charge density of the electron on the surface of a sphere with radius of $a_{0}$. It can be seen from Eq. 4 that the surface and point charge distribution of the electron around the nucleus in term of electrostatic energies are identical. Thus no energy is needed to transform the electron from point charge to surface charge and vice versa.

The speed of a wave traveling on a stretched string can be calculated as:

$$
v=\sqrt{\frac{F}{\mu}}
$$

where $\mathrm{F}$ is the tensional force and $\mu$ is the mass per unit length. Adopting this simplified model to a vibrating halo the velocity of the wave in the halo can be given as:

$$
v=\sqrt{\frac{\sigma}{\rho_{A}}}
$$

where $\sigma$ is the uniform surface stress in the halo and $\rho_{\mathrm{A}}$ is the area density. As a first approximation this plane velocity is used for the spherical surface of the electron shell in the Hydrogen atom (Fig. 2). The surface stress is then

$$
\sigma=\frac{\frac{e^{2}}{4 \pi \varepsilon_{o} a_{O}^{2}}}{2}=\frac{e^{2}}{2 \pi a_{o}}=.
$$

The area density of the electron halo is

$$
\rho_{A}=\frac{m_{e}}{4 \pi a_{o}^{2}}
$$


The velocity of the propagating wave is then

$$
v_{e}\left(a_{o}\right)=\sqrt{\frac{e^{2}}{4 \pi \varepsilon_{o} m_{e} a_{o}}}
$$

The wave velocity of the electron halo or surface charge electron is identical with the velocity of the point charge electron calculated from the Bohr model. Thus the proposed electron halo model using the laws of classical physics reproduces the features of the Bohr model. The additional atomic features, which are left unanswered by the previous models can also be explained by the surface charge electronic structure of the atom as follows.

1./ The symmetrical configuration and vibration of a surface charge electron halo around the nucleus should not emit radiation because of complete destructive interference. Thus at ground state and stationary state no emission should occur. This is consistent with the laws of classical electromagnetism. Thus, there is no need to invalidate part of the electromagnetic laws at atomic scale.

2./ There is no point charge, which orbit would become chaotic. The surface charge distribution of the electron halo is defined by the charge distribution of the nucleus. As long as the nucleus of the element is uniform the atoms will be identical. Thus the surface charge model is consistent with the stability and uniformity of the atoms.

3./ The mass center of the nucleus and the nucleus-electron system is the same in the surface charged electron halo model, which gives the right value for Bohr's radius.

4./ Disturbing the equilibrium of the uniform charge and mass distribution of the electron halo in the hydrogen atom will induces ripples on the surface. These ripples propagate as wave motion in the electron halo. The angular momentum of the propagating wave of this electron halo is $\mathrm{L}=\mathrm{m}_{\mathrm{e}} \mathrm{vr}=\mathrm{nh}$. Please note that not the electron is the one, which has the quantized 
angular momentum as was proposed by Bohr but rather the angular momentum of the propagating wave is quantized. In the same manner the speed of the electron in the Bohr's model should be replaced with the speed of the propagating wave. Thus, the surface charge distribution of the electron in the vicinity of the atom, gives a physical explanation for the quantized nature of the angular momentum.

Additionally, the angular momentum of the electron halo at the ground state is zero, which is consistent with experiments.

5./ The ionization energy of the surface charge electron distribution halo is exactly half of the energy required for removing a point charge electron (Fig. 3.). Thus the ionization energy of the surface charge electron is consistent with experiments.

6./ According to classical electromagnetism radiation is generated when charge accelerates. The orbiting point charge electron even at zero Kelvin should emit radiation, which is not observed. The surface charge electron halo does not emit radiation as long as there is no vibration or its vibration is symmetrical, which causes complete destructive interference. Radiation is emitted only when the propagating wave of the surface charge electron is asymmetric to the positive charge center of the nucleus. When the vibration switches from one symmetrical mode (stationary state) to another one, then during the transition period, the vibration becomes asymmetric. This asymmetric vibration results in electromagnetic radiation or the emission of the photon (Fig. 4).

7./ The atom built up from a practically stable nucleus surrounded by a vibrating electronic halo. It is suggested that the matter at atomic size scale behaves like a wave, when the physical interaction in the process is dominated by surface actions. If the interaction involves the entire bulk of the matter then the atom behaves as matter. 


\section{GENERALIZATION OF THE PROPOSED ATOMIC MODEL}

The proposed surface charge distribution of the electron in the vicinity of the nucleus should not be unique to the Hydrogen atom but rather be a universal feature of all the atoms. The generalization of the surface charge distribution of the electrons to all atoms is supported by the electron reactivity, which is defined as the average valence electron energy $(\mathrm{AVEE})^{25,26}$. Representing reactivity by AVEE shows that the electrons in the valence shell acts collectively and not individually. This collective behavior is only possible if the electrons from a unified electron shell. If the electrons would have single identity in the shell then electron reactivity should relate to the individual electrons with different energies in the shell. Experiments support a unified surface charge distribution in each of the electron shells of the atoms.

The charge of the nucleus in the Hydrogen atom is uniformly distributed resulting in a uniform surface charge of the electron in the Hydrogen atom. More complex nuclear structures proton charge distribution in the nucleus might not be uniform ${ }^{27,28}$. This non symmetrical nucleus charge distribution has effect on the electron shells resulting in a not completely uniform electron charge distribution.

\section{CONCLUSIONS}

Analyzing the pitfalls of previous atom models it is concluded that the point charge nature of the electron in the vicinity of the atom is unsustainable; therefore, it is postulated that the point charge electron transforms to surface charge when the electron is captured by the nucleus. No 
physical explanation for this process is offered; however, the proposed spherical surface charge electron halo model consistent with experiments as far as the author knowledge. The model allows explaining the emission and absorption of electromagnetic radiations of the atoms, the uniformity and stability of the atoms, the physics of the particle-wave duality nature of the matter, gives the correct value for the ground state angular momentum and the ionization energy of Hydrogen atom. Additionally the model consistent with classical electromagnetism and explains the physics how the laws of classical electromagnetism remains valid at atomic scale.

${ }^{1}$ E. Rutherford, Philosophical Magazine, Series 6, 669 (1911).

${ }^{2}$ J. Chadwick, Nature 129, 312 (1932).

${ }^{3}$ J. Chadwick Proceedings of the Royal Society of London 136, 692 (1932).

${ }^{4}$ N. Bohr Philosophical Magazine S. 6, 1, 476, 857 (1913).

${ }^{5}$ J.J. Balmer "Notiz ūber die Spektrallinien des Wasserstoffs" in Verhandlungen der Naturforschenden Gesellschaft in Basel 7, 548, 750; also in Annalen der Physik, 3rd ser. 25, 80 (1885).

${ }^{6}$ J.R. Rydberg The minutes of Society for Physics and Mathematics (Lund, Sweden) of November 5th, showing Rydberg's formula (1888).

${ }^{7}$ C. Baily European Physical Journal H 38, 1 (2013).

${ }^{8}$ A. Sommerfeld Annalen der Physik 51, 1 (1916)

${ }^{9}$ L. Broglie, Recherches sur la théorie des quanta (Researches on the quantum theory), Thesis, Paris, (1924), Ann. de Physique 3, 22 (1925).

${ }^{10}$ W. Heisenberg, Die Naturwissenschaften 14, 899 (1926)

${ }^{11}$ W. Heisenberg, Zeitschrift fur Physik 43, 172 (1927).

${ }^{12}$ C. Davisson, L.H. Germer, Phys. Rev. 30, 705 (1927).

${ }^{13}$ M. Born, P. Jordan, Zeitschrift für Physik 34, 858 (1925).

${ }^{14}$ M. Born, W. Heisenberg, P. Jordan, Zeitschrift für Phys. 35, 557 (1926).

${ }^{15}$ P.A.M. Dirac, Proc. R. Soc. London, Ser. A 109, 642 (1925). 
${ }^{16}$ P.A.M. Dirac, Proceedings of the Royal Society A: Mathematical, Physical and Engineering Sciences 117, 610 (1928).

${ }^{17}$ E. Schrödinger, Physical Review 28, 1049 (1926).

${ }^{18}$ M. Born, Zeitschrift für Physik 37, 863 (1926).

${ }^{19}$ M. Born, Zeitschrift für Phys. 38, 803 (1926).

${ }^{20}$ R.L. Mills, Phys. Essays 16, 443 (2003).

${ }^{21}$ P.J. Mohr, D.B. Newell, B.N.Taylor, arXiv:1507.07956 [physics.atom-ph] (2014).

${ }^{22}$ J.W. Nicholson, Mon. Not. Roy. Astr. Soc. 72, 677 (1912).

${ }^{23}$ NIST, Fundamental Physical Constants, CODATA (2014)

${ }^{24}$ D.R. Lide (Editor),Ionization potentials of atoms and atomic ions in Handbook of Chem. and Phys. $84^{\text {th }}$ edition, CRC Press, p. 10-211 (2004)

${ }^{25}$ L.C. Allen, J. Am. Chem. Soc. 111, 9003 (1989).

${ }^{26}$ L.C. Allen, Int. J. Quantum Chem. 49, 253 (1994).

${ }^{27}$ J. Garai, Int. J. Quantum Chem. 108, 667 (2008).

${ }^{28}$ J. Garai, arXiv:1101.4478v2 [physics.gen-ph] (2015). 
The point charge electron model of Hydrogen

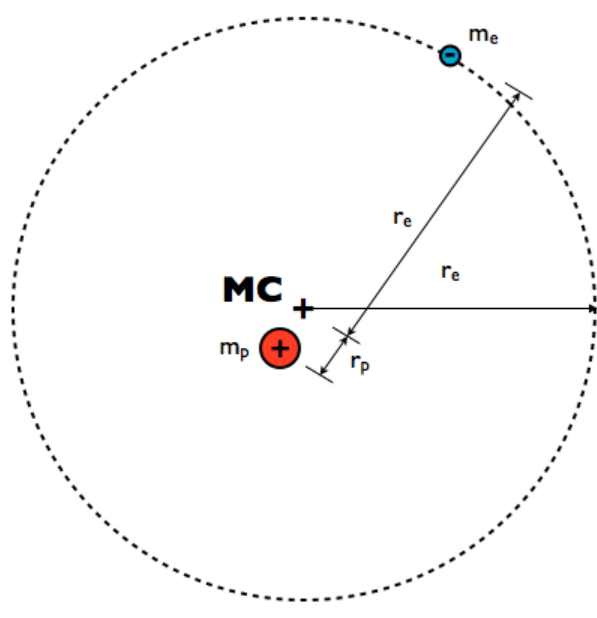

Not to scale!

FIG. 1. The point charge electron model of the Hydrogen is shown. The electron should orbit around the mass center of the proton-electron system. This effect introduces a small error into the Bohr radius, which has never been detected. The perfect correlation of the Bohr radius to other fundamental constants indicates that Bohr's assumption, the the mass center of the proton-electron system is the same as the mass center of the proton should remain valid.
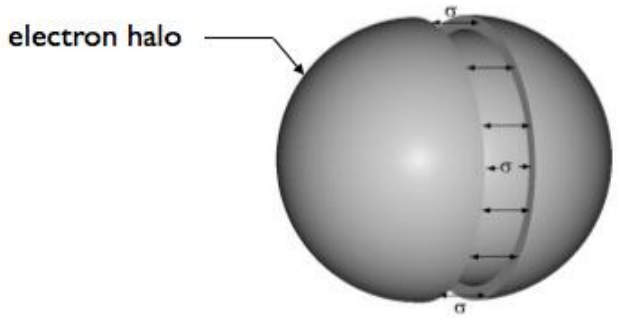

$$
\begin{gathered}
\sigma=\frac{e^{2}}{16 \pi^{2} \varepsilon_{o} a_{o}^{3}} \\
\rho_{A}=\frac{m_{e}}{4 \pi a_{o}^{2}}
\end{gathered}
$$

FIG. 2. The surface stress and the area density in the surface charge electron halo is shown. The spherical surface is substituted by a flat one in the calculation of wave velocity. 


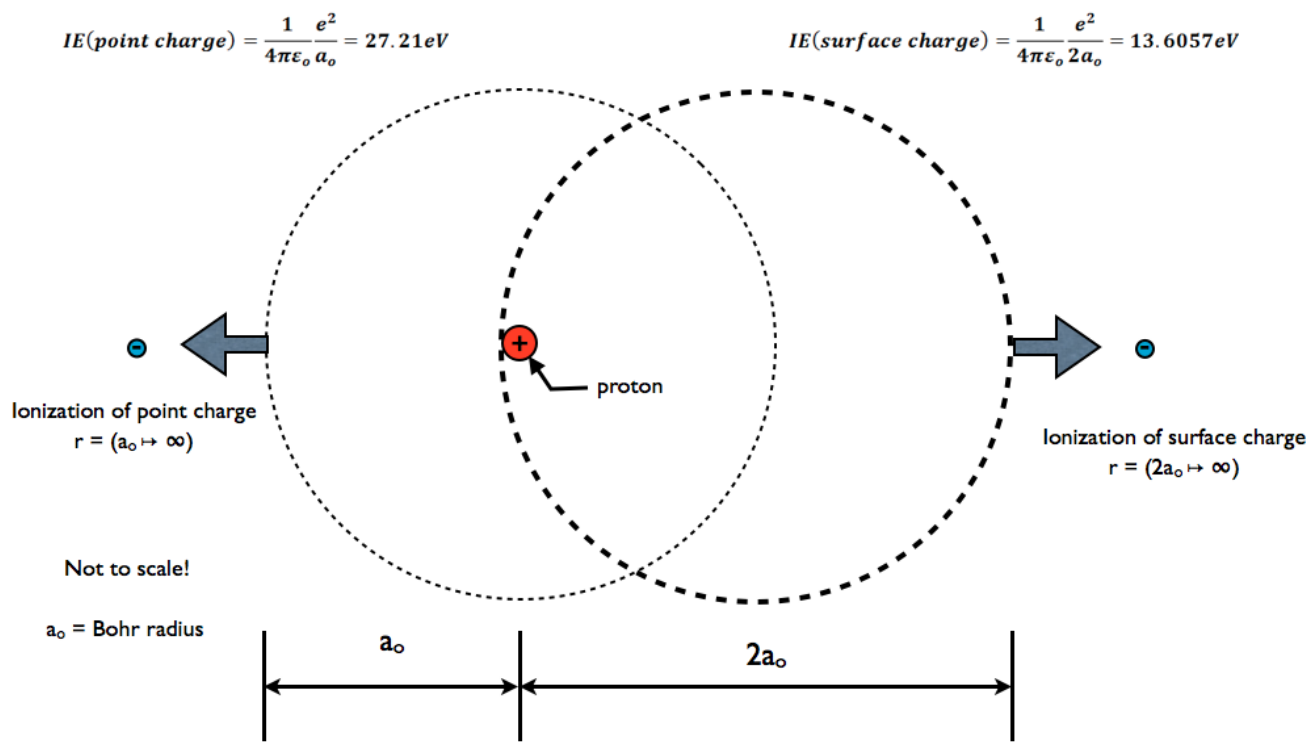

FIG. 3. The experimentally determined ionization energy of the Hydrogen atom is half of the one, which is required to remove the point charge electron from its ground state, which is from the Bohr's radius, to infinity. The electron of the Hydrogen atom in the proposed surface charge model transforms from surface charge to point charge at the distance of two Bohr's radius, which gives the experimentally determined value for the ionization energy. 


\section{Stationary State $\left[\mathrm{E}_{\mathrm{i}}\right]$ Symmetrical Waves No Emission}

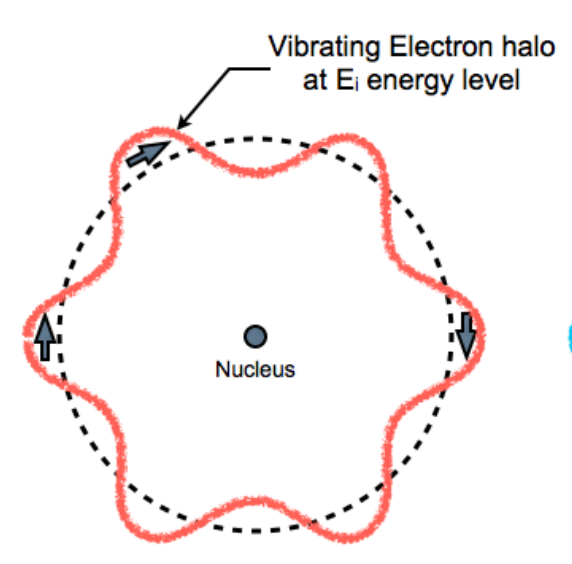

Transition Between Stationary States
Emission/Absorption Occurs

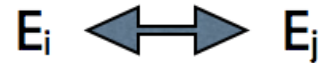

$\Delta E_{\text {photon }}=E_{j}-E_{i}$

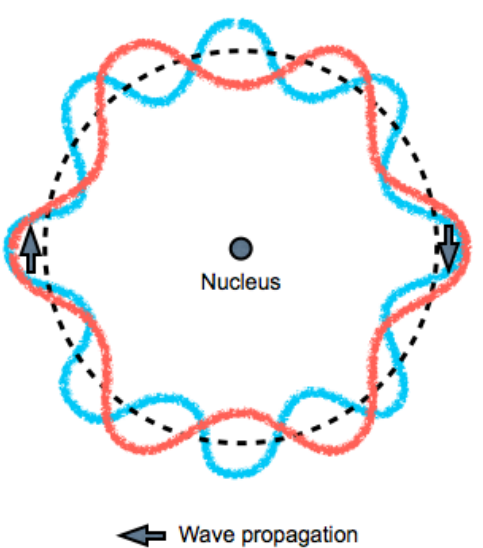

Stationary State $\left[\mathrm{E}_{\mathrm{j}}\right]$ Symmetrical Waves No Emission

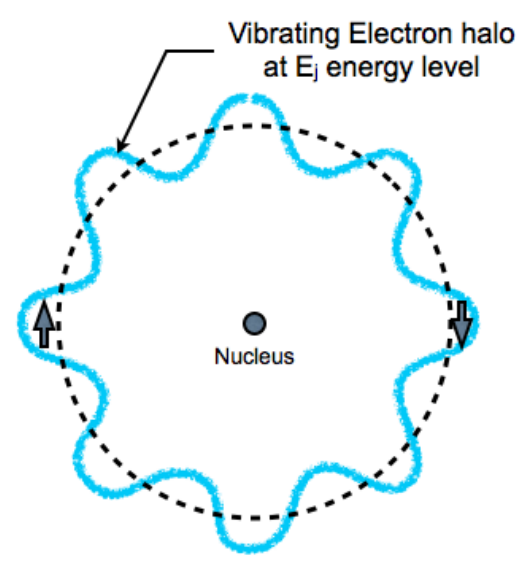

FIG. 4.

Schematic 2D figure shows the occurrence of the emission and the absorption of the electromagnetic radiation at atomic scale. The vibration of the electron halo around the nucleus is symmetrical at the stationary energy levels. Thus, no emission occurs resulting from destructive interference. However, when transition occurs between one symmetrical vibration state to the another symmetric one then electromagnetic radiation is emitted or absorbed. The uniform surface charge distribution of the electron explains the emission and absorption of photons without violating classical laws. Thus classical electromagnetism remains valid at atomic scale. 